\title{
Self-compassion and Adolescents' Positive and Negative Cognitive Reactions to Daily Life Problems
}

\author{
Peter Muris $\mathbb{1}^{1} \cdot{\text { Henry } \text { Otgaar }^{1} \cdot \text { Cor Meesters }^{1} \cdot \text { Amber Heutz }^{1} \cdot \text { Michelle van den Hombergh }}^{1}$
}

Published online: 11 February 2019

(c) The Author(s) 2019

\begin{abstract}
Objective To examine the relationship between self-compassion and positive and negative cognitive reactions to daily life problems in a sample of 117 adolescents aged 14-19 years.

Method Participants completed a survey containing the Self-Compassion Scale (SCS), the Adolescents' Reactions to Adversity Questionnaire - a self-developed scale for measuring cognitive responses to hypothetical scenarios describing daily life problems of young people (e.g., failing an important test, breaking up with a friend), and measures of neuroticism and self-esteem (control variables).

Results Self-compassion as indexed by the SCS total score was found to be associated with higher levels of positive cognitive reactions and lower levels of negative cognitive reactions. These relations remained present when controlling for neuroticism and self-esteem. Further analyses revealed a quite differential pattern of results for the compassionate (selfkindness, common humanity, and mindfulness) and uncompassionate (self-judgment, isolation, and over-identification) components of the SCS.

Conclusions This study indicates that self-compassion is a relevant construct for understanding adolescents' cognitive responses to daily problems and yields new evidence for our critical point that it is inappropriate to use the total selfcompassion score in a context of protection and resilience against life stress.
\end{abstract}

Keywords Self-compassion - Compassionate and uncompassionate self-responding Cognitive reactions to problems • Adolescents $\cdot$ Self-esteem $\cdot$ Neuroticism

During life, most people will experience adversity and difficulties from time to time. Some individuals are resilient and quickly adjust to such hardship, whereas other persons are more affected by the unpleasant events and prone to develop emotional problems. To understand individual differences in the way such life adversities are coped with, the psychological construct of self-compassion may be particularly relevant. Self-compassion has been defined as "being open to and moved by one's own suffering, experiencing feelings of caring and kindness toward oneself, taking an understanding, nonjudgmental attitude toward one's inadequacies and failures, and recognizing

Peter Muris

peter.muris@maastrichtuniversity.nl

1 Faculty of Psychology and Neuroscience, Department of Clinical Psychological Science, Maastricht University, P.O. Box 616, 6200 MD Maastricht, The Netherlands that such experiences are part of the common human experience" (Neff 2003a, p. 224). At a theoretical level, Neff (e.g., Neff et al. 2018b) views self-compassion as "the balance between increased compassionate and decreased uncompassionate self-responding to personal struggle" (p. 371), which involves three key elements on bi-polar ends: (1) being kind and supportive to oneself rather than harsh and judgmental (self-kindness as opposed to selfjudgment); (2) recognizing that such difficulties are a normal part of all humans' life rather than feeling isolated from other people as a result of one's imperfection (common humanity as opposed to isolation); and (3) keeping the personal suffering in rational awareness rather than becoming fully absorbed by one's problems (mindfulness as opposed to over-identification; see Neff 2003b).

A core assumption of self-compassion is that it promotes psychological resilience and enhances people's adaptation to life adversity and problems (Neff 2009). In line with this assumption, an increasing amount of empirical studies has shown that self-compassion is positively related to indices 
reflecting personal well-being (such as happiness and life satisfaction; Zessin et al. 2015) and negatively to measures tapping symptom levels of psychopathology (especially anxiety, depression, and stress) in both clinical and nonclinical samples (MacBeth and Gumley 2012). It is also assumed that these positive outcomes are the result of adaptive coping and emotion regulation strategies that are more likely to be deployed by people with high rather than low levels of self-compassion (Batts Allen and Leary 2010; Inwood and Ferrari 2018). Evidence for this assumption comes from various studies. First of all, correlational data as obtained in survey studies have demonstrated that selfcompassion appears to be positively related to adaptive coping strategies (e.g., active coping, planning, and positive reframing) and negatively associated with maladaptive strategies (e.g., behavioral disengagement and self-blame; Sirois et al. 2015). Furthermore, in a sample of patients with unipolar depression, Diedrich et al. (2017) found that the successful application of emotion regulation skills mediated the link between self-compassion and depressive symptoms. Finally, Leary et al. (2007) showed that self-reported (trait) and experimentally induced (state) levels of selfcompassion were associated with adaptive and positive emotional and cognitive reactions to unpleasant selfrelevant events.

In recent years, self-compassion has also been investigated in young people. Most of this research has been conducted with adolescents (Marsh et al. 2018; for an exception, see Sutton et al. 2018, who conducted their study in 8- to 12-year-old children), and the results are generally in line with what has been found in the adult literature, suggesting that self-compassion is a potent protective factor that is positively associated with adolescents' sense of wellbeing (e.g., Bluth and Blanton 2014) and negatively related to symptom levels of psychological distress and psychopathology (e.g., Bluth et al. 2017; see review and metaanalysis by Marsh et al. 2018). Whereas in adults at least a number of studies have investigated the link with coping/ emotion regulation as the hypothesized underlying mechanism for the adaptive function of self-compassion, this topic has been understudied in research with youths. One exception is a study by Bengtsson et al. (2016) who examined the structure of compassion-related constructs in 256 young adolescents aged 12 to 14 years. Aspects of selfcompassion were not only measured by means of the short version of the SCS (Raes et al. 2011) but also a number of other scales. Apart from a positive self-compassion component (which they labelled as 'self-focused perspectivetaking skills'), the authors found evidence for a second component, "which includes qualities that interfere with a compassionate state of mind, such as being harshly selfjudgmental or tormenting oneself with feelings of isolation and excessive worry" and as such representing "a tendency to engage in mental operations that generate of negative sense of self" (Bengtsson et al. 2016, p. 858). In other words, the results of this study suggest that there might be positive and negative cognitive coping processes underlying the construct of self-compassion. Further, a recent investigation by Muris et al. (2018, Study 2) explored relations between self-compassion and a broad variety of coping strategies. In this survey study of 130 high school students aged 15 to 19 years, prototypical indicators of selfcompassion (self-kindness, common humanity, and mindfulness) were positively associated with types of adaptive coping such as active tackling, social support seeking, and reassuring thoughts, but negatively linked to forms of maladaptive coping such as passive reacting and expression of emotion. Although this pattern was generally as expected, it should be noted that the coping measure as used by Muris et al. (2018; Study 2) included behavioral as well as cognitive coping strategies (Astor-Dubin and Hammen 1984). Given that self-compassion pre-eminently refers to an intra-psychic process (Bengtsson et al. 2016), it might be worthwhile to examine the relationship between selfcompassion and cognitive (coping) responses in more detail.

Thus, the current study further examined the link between self-compassion and cognitive (coping) responses in a sample of non-clinical adolescents. Participants completed Neff's (2003a) Self-Compassion Scale (SCS) to assess individual differences in self-compassion, and the Adolescents' Reactions to Adversity Questionnaire, a selfconstrued vignette-based scale for measuring positive and negative cognitive responses to a series of problems that might occur in the daily life of adolescents. It was hypothesized that adolescents with higher levels of selfcompassion would report higher levels of positive responses and lower levels of negative responses in times of adversity.

Apart from this general research purpose, a number of other issues were addressed in this study as well. First, in the past years, the validity of the SCS has received criticisms from several researchers. In keeping with Neff's (2003b) original definition of self-compassion, this scale not only contains items reflecting the three 'compassionate' components of self-kindness, common humanity, and mindfulness, but also includes items that measure their 'uncompassionate' counterparts of self-judgment, isolation, and over-identification. There has been quite some debate on the exact factor structure of the SCS: while evidence was obtained for a model with six correlated factors comprising the overall construct of self-compassion (e.g., Neff et al. 2017), support has also been found indicating that the scale can better be conceptualized as a two-factor measure with compassionate self-responding items loading on one and uncompassionate self-responding items loading on the other 
factor (e.g., Brenner et al. 2017; Costa et al. 2016; Lopez et al. 2015; Pfattheicher et al. 2017; Williams et al. 2014). Further, in a series of critical papers, it has been argued that the uncompassionate self-responding items of the SCS have much in common with psychopathology and so their inclusion in the total self-compassion score (by reversing scores on these items and summing them with scores on compassionate self-responding items) tends to inflate the (negative) relationship between self-compassion and psychopathological symptoms (Muris 2016; Muris and Petrocchi 2017; Muris et al. 2016b; Muris et al. 2019).

Moreover, compassionate and uncompassionate selfresponding components in the SCS have been demonstrated to relate in a different way to external constructs (e.g., Brenner et al. 2017; Brenner et al. 2018; Coroiu et al. 2018; Lopez et al. 2018; Neff et al. 2018a, 2018b). This can be linked to Gilbert's (2000) Theory of Social Mentalities, which proposes that there are two distinct but related processing systems that underlie people's patterns of cognition, affect, and behavior in response to interpersonal challenges: the safeness system and the threat-defense system. Activation of the safeness system will elicit thoughts, feelings, and actions that promote positive relationships with others (e.g., altruism, tolerance) and the self (e.g., self-compassion), while activation of the threatdefense system will prompt thoughts, feelings, and actions that mainly serve to reduce threat, which when directed externally can become manifest as avoidance and aggression towards others, and if directed inward exhibit themselves in various ways of uncompassionate self-responding (e.g., self-criticism, self-isolation, self-blame; see also Gilbert 2005). Thus, it can expected that SCS compassionate components will be predominantly associated with high levels of positive cognitive responses to daily problems in adolescents' lives, whereas SCS uncompassionate elements will be primarily associated with high levels of negative cognitive responses.

A second, additional issue that was investigated in this study concerned the link between self-compassion and two other psychological constructs that may be relevant for adolescents' adjustment to life stress (Muris et al. 2011) and hence also for their cognitive responses to problems. The first construct is self-esteem, which refers to a positive evaluation of one's worthiness as a person (Harter 1999), and the second is the basic personality trait of neuroticism, which can be defined as the individual's tendency to respond with negative emotion to (potentially) stressful life events (Ormel et al. 2004). Self-compassion appears to be positively correlated with self-esteem (e.g., Neff and Vonk 2009) and negatively linked to neuroticism (which seems mainly due to communalities between neuroticism and the uncompassionate self-responding components included in the SCS; Geiger et al. 2018; Pfattheicher et al. 2017). Given that high self-esteem has been shown to be associated with adaptive (problem-focused) coping and that neuroticism and low self-esteem have been found to be linked to maladaptive (emotion venting and disengagement) coping strategies (Carver and Conner-Smith 2010; Mullis and Chapman 2000), we explored the (unique) relations between selfcompassion and adolescents' cognitive (coping) reactions to hypothetical life stress, while controlling for self-esteem and neuroticism.

The third and final issue had to do with gender differences in self-compassion. There is a substantial amount of empirical studies showing that, already in adolescence, females (girls) display higher levels of emotional types of psychopathology than males (boys; e.g., Hankin et al. 1998; Lewinsohn et al. 1998) and as such one would also expect gender differences in factors playing a role in the etiology of such problems. As for individual difference variables, gender differences have been documented for coping styles (Hampel and Petermann 2005), personality traits (Costa et al. 2001), and self-related constructs (Muris et al. 2016a): in general, females/girls have been found to exhibit higher levels of maladaptive coping and unfavorable personality traits (i.e., neuroticism) but lower levels of protective selfrelated traits as compared to males/boys. In keeping with the latter, a recent meta-analysis by Yarnell et al. (2015) who evaluated gender differences in self-compassion using SCS data of 88 samples showed that females tend to report lower levels of self-compassion than males. However, the analysis only addressed gender differences in total selfcompassion and did not make a distinction between compassionate and uncompassionate self-responding. There are some indications that gender differences in self-compassion are mainly due to differences in the uncompassionate components of the SCS (i.e., girls/females scoring higher on self-judgment, isolation, and over-identification than males/ boys) and that both genders do not differ with regard to the compassionate components (i.e., self-kindness, common humanity, and mindfulness; e.g., Bluth and Blanton 2014), which is a hypothesis that will be further investigated in the present study.

\section{Method}

\section{Participants}

Participants were 117 high school students of Raayland College in Venray, The Netherlands. There were 42 boys and 75 girls aged between 14 and 19 years, with a mean age of 15.82 years $(S D=0.87)$. All students followed preuniversity education (in Dutch: Voortgezet Wetenschappelijk Onderwijs) and were in the $9^{\text {th }}(16.2 \%), 10^{\text {th }}(40.2 \%)$, or 11 th $(43.6 \%)$ grade. Due to school constraints, no further 
information was available regarding the ethnic and socioeconomic background of the students.

\section{Procedure}

After the board of Raayland College had given permission to conduct the study at school, all students $(N=330)$ were approached during mentor classes. They first received oral information about the study and - if interested - they were given an information letter along with a consent form, which had to be signed by them and their parents and returned to the researchers via the teacher. One-hundredand-seventeen students eventually did so, yielding a response percentage of $35.5 \%$. The participating students completed the survey - which contained four questionnaires - during regular classes at school. This assessment took about $20 \mathrm{~min}$ and was supervised by the fourth and fifth author, who handled questions and ensured confidential responding. As a compensation for their participation, 10 cinema tickets were raffled among the participating students. The study was approved by the Ethical Review Committee of Psychology and Neuroscience at Maastricht University (reference number: Master-192-15-04-2018).

\section{Materials}

\section{Self-Compassion Scale}

Individual differences in self-compassion were measured with the SCS (Neff 2003a). This scale consists of 26 items representing compassionate and uncompassionate ways of self-responding during times of adversity. As noted in the introduction, the compassionate components are selfkindness (5 items; e.g., "When I'm going through a very hard time, I give myself the caring and tenderness I need"), common humanity (4 items; e.g., "When I'm down and out, I remind myself that there are lots of other people in the world feeling like I am"), and mindfulness (4 items; e.g., "When something painful happens, I try to take a balanced view of the situation"), while the uncompassionate components reflect their counterparts of self-judgment (5 items; e.g., "When I see aspects of myself that I don't like, I get down on myself"), isolation (4 items; e.g., "When I fail at something that is important to me, I tend to feel alone in my failure"), and over-identification (4 items; e.g., "When something painful happens, I tend to blow the incident out of proportion"). Items are scored on a 5-point Likert scale with $1=$ almost never and $5=$ almost always) and can be combined to generate a total score (which includes the reversed scores of uncompassionate items) and scores for the separate components (as advocated by Neff and colleagues; e.g., Neff 2016) as well as the two components of compassionate and uncompassionate self-responding (CSR and USR; see Brenner et al. 2017; Costa et al. 2016; Lopez et al. 2015; Pfattheicher et al. 2017; Williams et al. 2014). There is good evidence for the basic psychometric qualities of the scale (Neff 2003a). In this study, Cronbach's alphas for various SCS scales ranged between 0.63 (common humanity) and 0.90 (uncompassionate self-responding).

\section{Adolescents' Reactions to Adversity Questionnaire}

The Adolescents' Reactions to Adversity Questionnaire (ARAQ) is a self-construed scale for measuring cognitive responses to 10 hypothetical scenarios describing typical problems that may occur in the daily life of young people (Byrne and Mazanov 2002; Wright et al. 2010): failing an important test, blocking during a presentation, coming too late at school because of oversleeping, being the cause of friction between parents, forgetting a friend's birthday, a friend doesn't show up at an appointment, blundering during sports, bad music performance in front of an audience, breaking up with a friend, and accidentally damaging a friend's property. Each scenario was followed by six cognitive responses for which participants had to indicate on a 4-point scale to what extent they would be applying to them ( $1=$ not all, $4=$ very much). Three of the cognitive responses were positive as they referred to humor (i.e., "This is kind of funny"), optimism (i.e., "In the long run, it will be okay"), and perspective taking (i.e., "This can happen to anybody"), while three cognitive responses were negative and referred to dramatizing (i.e., "This is awful"), putting oneself down (i.e., "I am a loser"), and shame (i.e., "I feel very ashamed"). Scores can be combined for each type of cognitive response (range: 10-40) and for negative and positive responses combined (range: 30-120).

\section{Self-Perception Profile for Children}

Self-esteem was measured by means of Harter's (1985) Self-Perception Profile for Children (SPPC) that can be used in child as well as adolescent populations. The scale contains 36 items that can be allocated to five specific domains of self-esteem, namely academic competence, social acceptance, athletic competence, physical appearance, and behavioral conduct, as well as global self-worth. Originally, each SPPC item consists of two opposite descriptions, e.g., "Some children often forget what they have learned" but "Other children are able to remember all things easily". Children have to choose the description that best fits and then indicate whether the description is somewhat true or very true for them. In the present study, a different version of the SPPC was used: positive descriptions were formulated from the young person's perceptive (e.g., "I am able to remember all things easily"), and participants had to indicate to what extent these descriptions were true for them 
( $1=$ not at all true, $4=$ very true $).$ A total self-esteem score was obtained by summing across all items (range: 36-144). Psychometric evaluations of the SPPC (including the modified version that was used in the current study) have shown that this scale provides a reliable and valid index of self-esteem in children and adolescents (Harter 1985; Muris et al. 2003). In the current investigation, the internal consistency coefficient of the SPPC was excellent $(\alpha=0.90)$.

\section{Big Five Questionnaire for Children}

Neuroticism was assessed using a subscale of the Big Five Questionnaire for Children (BFQ-C; Barbaranelli et al. 2003), which is a self-report questionnaire for measuring the five higher-order factors of personality in youth from the age of 8 years. The neuroticism scale of the BFQ-C consists of 15 items (e.g., "I get nervous for silly things") that have to be scored on a five-point Likert scale ranging from $1=$ almost never to $5=$ almost always. Individual item scores are combined to yield a total neuroticism score ranging from 15 to 60 , with higher scores reflecting higher levels of this personality trait. Support has been found for the psychometric qualities of the BFQ-C, and the neuroticism subscale in particular (Barbaranelli et al. 2003; Muris et al. 2005). In the present study, the BFQ-C neuroticism scale had a Cronbach's alpha of 0.74 .

\section{Results}

\section{Factor Structure and Reliability of the ARAQ}

Because the ARAQ was a self-construed scale that was used for the first time in this study, we first conducted some tests to explore its structural validity and reliability. A principal components analysis (with oblimin rotation) performed on the six cognitive responses subscales yielded two factors with eigenvalues greater than 1 (i.e., 3.06 and 1.08), which together explained $69.00 \%$ of the variance. Inspection of the two-factor solution indicated that dramatizing, putting oneself down, and shame constituted the first factor of negative cognitive responses (factor loadings being 0.81, 0.80 , and 0.88 , respectively), while humor, optimism, and perspective taking loaded positively on the second factor of positive cognitive responses (factor loadings being 0.42 , 0.92 , and 0.87 , respectively). Some secondary loadings were found but these were all negative (i.e., humor and perspective taking loaded -0.60 and -0.54 on the negative cognitive responses factor), which also expressed itself in a negative correlation of -0.39 between both factors. Further, when using a different statistical approach (i.e., principal axis factoring), similar results were found. That is, the six ARAQ subscales again split into two factors: one factor containing the negative cognitive responses of dramatizing, putting oneself down, and shame (factor loadings being $0.98,0.69$, and 0.66 ) and the other factor consisting of the positive cognitive responses of humor, optimism, and perspective taking (factor loadings being $0.21,0.87$, and 0.70 ). Only humor showed a substantial secondary (negative) loading of -0.38 . The internal consistency reliability of the ARAQ was good. Cronbach's alphas were 0.89 for positive cognitive responses, 0.93 for negative cognitive responses, and between 0.74 and 0.89 for specific cognitive responses subscales (Table 1).

\section{Gender Differences}

A number of significant gender differences were noted (Table 1). More precisely, boys displayed higher total selfcompassion scores than girls $[t(115)=3.04, p<0.01$, Cohen's $d=0.61$ ], although a closer inspection of scores on various self-components revealed that this was primarily due to differences in uncompassionate self-responding $[t$ $(115)=3.87, \mathrm{p}<0.001, d=0.75]$ and this showed itself in all three components of self-judgment $[t(115)=2.35, p<$ $0.05, d=0.46]$, isolation $[t(115)=3.82, p<0.001, d=$ $0.74]$, and over-identification $[t(115)=4.03, p<0.001, d=$ 0.77]. Further, girls also reported higher levels of negative cognitive reactions on the ARAQ $[t(115)=4.22, p<0.001$, $d=0.79]$ than boys, and this difference was observed for dramatizing $[t(115)=3.78, p<0.001, d=0.68]$, putting oneself down $[t(115)=2.96, p<0.01, d=0.57]$, and shame $[t(115)=3.86, p<0.001, d=0.73]$. Finally, girls displayed higher levels of neuroticism $[t(115)=3.97, p<0.001, d=$ $0.78]$, while boys exhibited higher scores of self-esteem $[t$ $(115)=4.81, p<0.001, d=0.93]$. Given these differences, it was decided to control for gender in all further analyses.

\section{Relations Between Self-compassion and Cognitive Reactions}

The correlations (corrected for gender) between SCS scores and cognitive reactions as measured by the ARAQ are displayed in Table 2. Note that the SCS total score was statistically significantly, positively correlated with positive cognitive reactions $(r=0.40, p<0.001)$ and especially optimism $(r=0.32, p=0.001)$ and perspective taking $(r=$ $0.45, p<0.001)$, while statistically significant negative correlations were observed with negative cognitive reactions $(r=-0.50, p<0.001)$ and in particular putting oneself down $(r=-0.51, p<0.001)$ and shame $(r=-0.49$, $p<0.001)$.

When looking at separate correlations of SCS compassionate and uncompassionate self-responding (CSR and USR) to cognitive reactions as measured by the ARAQ (Table 2), it was found that both CSR and USR were 
Table 1 Descriptive statistics (means, standard deviations, gender differences, and internal consistency coefficients) for various questionnaires

\begin{tabular}{|c|c|c|c|c|}
\hline & $\begin{array}{l}\text { Total sample } \\
(N=117)\end{array}$ & Boys $(n=42)$ & Girls $(n=75)$ & $\begin{array}{l}\text { Cronbach's } \\
\text { alpha }\end{array}$ \\
\hline SCS Total Score & $75.00(13.44)$ & $79.88(11.00)$ & $72.27(13.96)^{*}$ & 0.88 \\
\hline $\begin{array}{l}\text { SCS Compassionate Self- } \\
\text { responding }\end{array}$ & $34.84(7.32)$ & $35.64(6.93)$ & $34.39(7.54)$ & 0.82 \\
\hline Self-kindness & $13.43(3.44)$ & $13.93(3.21)$ & $13.15(3.56)$ & 0.74 \\
\hline Common Humanity & $9.91(2.85)$ & $9.60(2.74)$ & $10.08(2.91)$ & 0.63 \\
\hline Mindfulness & $11.50(2.85)$ & $12.12(2.94)$ & $11.16(2.77)$ & 0.69 \\
\hline $\begin{array}{l}\text { SCS Uncompassionate Self- } \\
\text { responding }\end{array}$ & $37.91(9.73)$ & $33.52(8.89)$ & $40.37(9.35)^{*}$ & 0.90 \\
\hline Self-judgment & $14.98(3.95)$ & $13.86(3.60)$ & $15.61(4.02)^{*}$ & 0.81 \\
\hline Isolation & $11.17(3.64)$ & $9.55(3.22)$ & $12.08(3.60)^{*}$ & 0.79 \\
\hline Over-identification & $11.76(3.51)$ & $10.12(3.35)$ & $12.68(3.27)^{*}$ & 0.76 \\
\hline $\begin{array}{l}\text { ARAQ Positive Cognitive } \\
\text { Reactions }\end{array}$ & $76.52(12.88)$ & $77.83(15.56)$ & $75.79(11.15)$ & 0.89 \\
\hline Humor & $18.68(6.05)$ & $20.21(7.10)$ & $17.83(5.24)$ & 0.85 \\
\hline Optimism & $31.09(5.55)$ & $30.67(6.29)$ & $31.32(5.12)$ & 0.74 \\
\hline Perspective Taking & $26.75(4.82)$ & $26.95(5.24)$ & $26.64(4.60)$ & 0.84 \\
\hline $\begin{array}{l}\text { ARAQ Negative Cognitive } \\
\text { Reactions }\end{array}$ & $71.75(15.66)$ & $64.12(16.61)$ & $76.03(13.41)^{*}$ & 0.93 \\
\hline Dramatizing & $30.36(5.50)$ & $27.93(6.62)$ & $31.72(4.23)^{*}$ & 0.85 \\
\hline Putting Oneself Down & $18.70(6.49)$ & $16.40(6.15)$ & $19.99(6.36)^{*}$ & 0.89 \\
\hline Shame & $22.69(6.45)$ & $19.79(6.47)$ & $24.32(5.88)^{*}$ & 0.85 \\
\hline SPPC Self-esteem & $95.14(13.64)$ & 102.57 (12.16) & $90.97(12.68)^{*}$ & 0.90 \\
\hline BFQ-C Neuroticism & $23.47(5.14)$ & $21.10(4.45)$ & $24.80(5.04)^{*}$ & 0.74 \\
\hline
\end{tabular}

SCS Self-Compassion Scale, ARAQ Adolescents' Reactions to Adversity Questionnaire, SPPC SelfPerception Profile for Children, $B F Q-C$ Big Five Questionnaire for Children

*Significant gender difference at $p<0.05$

Table 2 Correlations (corrected for gender) between various SCS scores and measures of cognitive reactions, self-esteem, and neuroticism

\begin{tabular}{|c|c|c|c|c|c|c|c|c|c|c|c|}
\hline & \multicolumn{9}{|l|}{ SCS } & \multirow{2}{*}{$\begin{array}{l}\text { SPPC } \\
\text { SE }\end{array}$} & \multirow{2}{*}{$\begin{array}{l}\text { BFQ-C } \\
\mathrm{N}\end{array}$} \\
\hline & Total & CSR & SK & $\mathrm{CH}$ & M & USR & SJ & I & $\mathrm{OI}$ & & \\
\hline ARAQ Positive Cognitive Reactions & $0.40 *$ & $0.34 *$ & $0.27 *$ & 0.20 & $0.33 *$ & $-0.31^{*}$ & $-0.28 *$ & -0.24 & $-0.29 *$ & 0.25 & -0.11 \\
\hline Humor & 0.20 & 0.11 & 0.05 & 0.13 & 0.11 & -0.21 & -0.15 & -0.17 & -0.22 & 0.05 & 0.10 \\
\hline Optimism & $0.32 *$ & $0.35 *$ & $0.32 *$ & 0.17 & $0.35 *$ & -0.17 & -0.20 & -0.12 & -0.13 & $0.28 *$ & -0.16 \\
\hline Perspective Taking & $0.45^{*}$ & $0.35 *$ & $0.31 *$ & 0.19 & $0.34 *$ & $-0.37 *$ & $-0.32 *$ & $-0.29 *$ & $-0.35 *$ & $0.29 *$ & -0.23 \\
\hline ARAQ Negative Cognitive Reactions & $-0.50 *$ & -0.23 & $-0.32 *$ & -0.04 & -0.16 & $0.54 *$ & $0.45^{*}$ & $0.44 *$ & $0.51 *$ & $-0.43^{*}$ & $0.39 *$ \\
\hline Dramatizing & -0.23 & -0.12 & -0.18 & 0.01 & -0.11 & 0.24 & 0.15 & 0.18 & $0.30 *$ & -0.16 & 0.22 \\
\hline Putting Oneself Down & $-0.51^{*}$ & $-0.27 *$ & $-0.35^{*}$ & -0.07 & -0.19 & $0.51 *$ & $0.44^{*}$ & $0.45^{*}$ & $0.44 *$ & $-0.47 *$ & $0.40^{*}$ \\
\hline Shame & $-0.49 *$ & -0.17 & -0.26 & -0.04 & -0.10 & $0.57 *$ & $0.51 *$ & $0.45 *$ & $0.51 *$ & $-0.40^{*}$ & $0.34 *$ \\
\hline SPPC Self-esteem & $0.44 *$ & $0.35 *$ & $0.43^{*}$ & 0.11 & 0.26 & $-0.35^{*}$ & $-0.39 *$ & $-0.32 *$ & -0.18 & - & $-0.36^{*}$ \\
\hline BFQ-C Neuroticism & $-0.52 *$ & -0.24 & $-0.35^{*}$ & -0.05 & -0.14 & $0.56 *$ & $0.46^{*}$ & $0.47 *$ & $0.53 *$ & $-0.36^{*}$ & - \\
\hline
\end{tabular}

SCS Self-Compassion Scale, CSR Compassionate Self-Responding, SK Self-Kindness, CH Common Humanity, M Mindfulness, USR Uncompassionate Self-Responding, SJ Self-Judgment, I Isolation, OI Over-Identification, SPPC Self-Perception Profile for Children, SE Selfesteem, $B F Q-C$ Big Five Questionnaire for Children, $N$ Neuroticism, ARAQ Adolescents' Reactions to Adversity Questionnaire ${ }^{*} p<0.05 / 10$ (i.e., Bonferroni correction; for reasons of clarity, significance is only indicated using this stringent criterion)

statistically significantly associated with positive cognitive reactions. As expected, the link between CSR and positive cognitive reactions was positive $(r=0.34, p<0.001)$ and mainly carried by statistically significant correlations between the SCS components of self-kindness and mindfulness and ARAQ optimism and perspective taking, 
whereas that between USR and positive cognitive reactions was negative $(r=-0.31, p=0.001)$ and predominantly attributable to the statistically significant correlations between all three uncompassionate SCS components and ARAQ perspective taking.

Correlations with ARAQ negative cognitive reactions showed a differential pattern for CSR and USR. That is, whereas CSR was less convincingly negatively correlated with negative cognitive reactions (for the total score: $r=$ $-0.23, p=0.01$ ) with self-kindness being the only component showing a statistically significant negative correlation to putting oneself down, USR was substantially positively correlated with negative cognitive reactions (for the total score: $r=0.54, p<0.001)$ and this link was carried by all three uncompassionate components being statistically significantly positively related to putting oneself down and shame.

\section{Relations Between Self-compassion and Self- esteem/neuroticism}

Correlations (again corrected for gender) between SCS scores and SPPC self-esteem and BFQ-C neuroticism are also displayed in Table 2. As can be seen, the SCS total score was statistically significantly positively correlated with self-esteem $(r=0.44, p<0.001)$. The correlation between CSR and self-esteem was positive $(r=0.35, p<$ 0.001 ) and mainly carried by self-kindness, whereas that between USR and self-esteem was negative $(r=-0.35, p<$ 0.001 ) and predominantly produced by the components of self-judgment and isolation.

The SCS total score was statistically significantly negatively correlated with neuroticism $(r=-0.52, p<0.001)$. The correlation between CSR and neuroticism was weak $(r=-0.24, p=0.01)$ and in most cases non-significant; only the component of self-kindness showed a more substantial, statistically significant negative link $(r=-0.35$, $p<0.001)$. In contrast, there was a substantial, positive and statistically significant correlation between USR and neuroticism (for the total score: $r=0.56, p<0.001$ ) and this link was found for all three USR components (i.e., selfjudgment, isolation, and over-identification).

\section{Unique Relations Between Self-compassion and Cognitive Reactions}

As shown in the right columns of Table 2, SPPC selfesteem and BFQ-C neuroticism (which were negatively correlated to each other: $r=-0.36$ ) also displayed statistically significant associations with adolescents' cognitive reactions to the hypothetical scenarios as listed in the ARAQ. More precisely, self-esteem correlated positively with optimism and perspective taking, while negative correlations were noted with negative cognitive reactions in general $(r=-0.43)$ and putting oneself down and shame in particular. Neuroticism was positively correlated with negative cognitive reactions $(r=0.39)$ and especially with putting oneself down and shame.

To explore the unique contribution of self-compassion for adolescents' cognitive reactions to daily life problems, regression analyses were performed with various SCS scores as predictors in which we also controlled for gender, neuroticism, and self-esteem. In the first series of regression analyses, the unique relation between self-compassion and positive cognitive reactions was explored (see top panel of Table 3). When using the total SCS score (i.e., Model 1), self-compassion made a statistically significant positive contribution to positive cognitive reactions $(\beta=0.44, p<$ 0.001 ), whereas neuroticism and self-esteem did not. When

Table 3 Main results of the regression analyses predicting adolescents' positive and negative coping responses to daily life problems from various SCS scores, gender, SPPC self-esteem, and BFQ-C neuroticism

\begin{tabular}{|c|c|c|c|c|}
\hline & $B$ & $S E$ & $\beta$ & $R^{2}$ \\
\hline \multicolumn{5}{|l|}{ ARAQ Positive Cognitive Reactions } \\
\hline Model 1: $F(4,112)=6.39, p<0.001$ & & & & 0.19 \\
\hline Gender & 1.09 & 2.55 & 0.04 & \\
\hline SPPC Self-esteem & 0.12 & 0.10 & 0.13 & \\
\hline BFQ-C Neuroticism & 0.39 & 0.27 & 0.16 & \\
\hline SCS Total Score & 0.42 & 0.11 & $0.44 * *$ & \\
\hline Model 2: $F(5,111)=5.15, p<0.001$ & & & & 0.19 \\
\hline Gender & 1.17 & 2.59 & 0.04 & \\
\hline SPPC Self-esteem & 0.12 & 0.10 & 0.13 & \\
\hline BFQ-C Neuroticism & 0.38 & 0.28 & 0.15 & \\
\hline CSR & 0.44 & 0.16 & $0.25 *$ & \\
\hline USR & -0.39 & 0.15 & $-0.30 *$ & \\
\hline \multicolumn{5}{|l|}{ ARAQ Negative Cognitive Reactions } \\
\hline Model 1: $F(4,112)=19.56, p<0.001$ & & & & 0.41 \\
\hline Gender & 4.36 & 2.64 & 0.13 & \\
\hline SPPC Self-esteem & -0.27 & 0.10 & $-0.24 *$ & \\
\hline BFQ-C Neuroticism & 0.43 & 0.28 & 0.14 & \\
\hline SCS Total Score & -0.37 & 0.11 & $-0.32 * *$ & \\
\hline Model 2: $F(5,111)=17.96, p<0.001$ & & & & 0.42 \\
\hline Gender & 3.15 & 2.60 & 0.10 & \\
\hline SPPC Self-esteem & -0.30 & 0.10 & $-0.26^{*}$ & \\
\hline BFQ-C Neuroticism & 0.22 & 0.28 & 0.07 & \\
\hline CSR & -0.02 & 0.16 & -0.01 & \\
\hline USR & 0.65 & 0.15 & $0.41 * *$ & \\
\hline
\end{tabular}

CS Self-Compassion Scale, CSR Compassionate Self-Responding, USR Uncompassionate Self-Responding, ARAQ Adolescents' Reactions to Adversity Questionnaire, SPPC Self-Perception Profile for Children, $B F Q-C$ Big Five Questionnaire for Children

${ }^{*} p<0.05, * * p<0.05 / 4$ or 5 (i.e., Bonferroni correction) 
employing CSR and USR scores as separate predictors (i.e., Model 2), both compassionate $(\beta=0.25, p<0.01)$ and uncompassionate $(\beta=-0.30, p=0.01)$ self-responding accounted for a unique proportion of the variance in positive cognitive reactions. When including the six SCS subscales in the model, it was found that only the components of mindfulness $(\beta=0.32, p<0.05)$ and self-judgment $(\beta=$ $-0.27, p<0.05$ ) made unique statistically significant contributions.

The second series of regression analyses examining unique links between self-compassion and negative cognitive reactions (see bottom panel of Table 3) showed that when using the SCS total score (i.e., Model 1), only selfcompassion $(\beta=-32, p<0.001)$ and self-esteem $(\beta=$ $-0.24, p<0.05)$ made statistically significant negative contributions. When employing the CSR and USR scores as predictor variables (i.e., Model 2), it was found that besides self-esteem $(\beta=-0.26, p<0.05)$ only uncompassionate self-responding $(\beta=0.41, p<0.001)$ accounted for a significant proportion of the variance in negative cognitive responses. Note that the beta value was positive, which indicates that higher levels of uncompassionate selfresponding were associated with higher levels of negative cognitive response to daily life problems. A final regression analysis with the six SCS subscales as predictors revealed that it was mainly the uncompassionate self-responding component of over-identification $(\beta=0.39, p<0.001)$ that carried this effect.

Given that there was a significant, negative correlation between the negative and positive cognitive reactions as measured by the ARAQ, all of the above regression analyses were repeated while controlling for this relationship. Self-compassion as indexed by the SCS total score remained a significant predictor of positive cognitive reactions $(\beta=0.28, p<0.01)$, and this was mainly due to the unique contribution of CSR $(\beta=0.25, p<0.01)$. When predicting negative cognitive reactions, the SCS total score did not explain a significant proportion of the variance $(\beta=$ $-0.16, p=0.07)$, but here the contribution of USR was still found to be significant $(\beta=0.30, p=0.001)$.

\section{Discussion}

Self- compassion refers to a positive way of dealing with oneself when confronted with personal difficulties and adversity in life. Self-compassion consists of three key components: (1) being kind and understanding to oneself (rather than being critical and judgmental); (2) realizing that personal difficulties are a common phenomenon in the life of all people (rather than feeling isolated in one's suffering); and (3) maintaining a balanced and accepting view on the problem (rather than becoming fully absorbed by it; Neff 2003a, 2003b). In the past years, an increasing number of studies have been conducted to demonstrate the relevance of self-compassion within the context of mental illness and psychopathology. Although most of this research has relied on cross-sectional and correlational designs, a clear negative association between self-compassion and symptoms of anxiety, depression, and stress has been detected in these studies (MacBeth and Gumley 2012), which is in line with the hypothesized protective nature of the self-compassion construct.

While it is assumed that the positive effects of selfcompassion are based on the deployment of adaptive cognitive coping/emotion regulation strategies (Batts Allen and Leary 2010; Inwood and Ferrari 2018), relatively few studies have addressed this topic empirically and this is especially the case in young people. One exception was a recent study by Muris et al. (2018, Study 2) which demonstrated that self-compassion is positively associated with adaptive coping strategies such as active tackling, social support seeking, and reassuring thoughts, but negatively related to maladaptive coping such as passive reacting and expression of emotion. Because self-compassion pertains to an intra-psychic process, the present study was focused on its relationships to cognitive coping responses. Based on the results of previous investigations with adolescents (Bengtsson et al. 2016; Muris et al. 2018, Study 2) and research conducted in adult populations (Diedrich et al. 2017; Leary et al. 2007; Sirois et al. 2015), we hypothesized that self-compassion would be positively linked to positive cognitive reactions, whereas negative associations were anticipated with negative cognitive reactions. Our results mirrored these predictions and demonstrated that higher levels of self-compassion as measured with the SCS total score were accompanied by higher levels of humor, optimism, and perspective taking, which all can be viewed as correlates of cognitive restructuring (Ayers et al. 1996), positive reappraisal (Ebata and Moos 1991), or positive self-talk (Hogendoorn et al. 2010). Furthermore, higher levels of self-compassion as indexed by the SCS total score were associated with lower levels of dramatizing, putting oneself down, and shame, which can be considered as venting of emotion (Phelps and Jarvis 1994), self-blame (O'Brien et al. 1997), or negative self-talk (Schniering and Rapee 2002).

Although the above described findings are in line with our main hypothesis, one important comment needs to be made. In the past years, we have made critical remarks regarding the use of the SCS total score as an index of selfcompassion (Muris 2016; Muris and Petrocchi 2017; Muris et al. 2016b, 2018, 2019). The central point of the critique is that the SCS incorporates compassionate (CSR; i.e., selfkindness, common humanity, and mindfulness) and uncompassionate (USR; i.e., self-judgment, isolation, and 
over-identification) components of self-responding. Evidence for the existence of two separate components of selfcompassion has been obtained in factor-analytic studies of the SCS conducted by various research groups (Brenner et al. 2017; Costa et al. 2016; Lopez et al. 2015; Pfattheicher et al. 2017; Williams et al. 2014) as well as in the investigation by Bengtsson et al. (2016) who examined structural models of compassion-related traits using data collected by means of multiple scales. The CSR component clearly reflects protective mechanisms, but the USR component unambiguously represents features of vulnerability (Muris and Petrocchi 2017), which of course is difficult to reconcile with the premises of a positive psychology construct. The present study adds further evidence showing that CSR and USR are quite different in nature (see also Brenner et al. 2017, 2018; Coroiu et al. 2018; Lopez et al. 2018; Muris et al. 2018; Neff et al. 2018b). That is, as anticipated, compassionate self-responding was positively related to positive cognitive reactions, whereas uncompassionate selfresponding was mainly positively associated with negative cognitive reactions.

We do not want to fully reiterate our critique that the inclusion of uncompassionate self-responding items in the SCS is problematic and even redundant, especially if one is interesting in studying self-compassion as a protective factor within the context of psychopathology. However, for those readers who are not familiar with our stance an example of a recent study is given in which these problems become particularly evident. This investigation was conducted by Gill et al. (2018) to examine the relation between self-compassion and social anxiety in an adolescent population. The researchers found that "self-compassion was inversely correlated with social anxiety with a large effect size $(r=-0.551)$ " and that this finding suggests that "selfcompassion could be an important factor in the development of social anxiety" (p. 163). Yet, when looking at the correlations between SCS subscales and social anxiety, it becomes clear that the inverse correlation between selfcompassion and social anxiety was almost fully carried by the (reversely scored) uncompassionate components of selfjudgment, isolation, and over-identification. Although noted by Gill et al. (2018), they discarded this finding because "no specific hypotheses were set on the individual subscales of the SCS" (p. 169), which is at the least peculiar as there is a host of studies showing that self-criticism (cf. self-judgment; Kopala-Sibley et al. 2013), loneliness (cf. isolation; Lim et al. 2016), and ruminative thinking (cf. over-identification; Penney and Abbott 2014) are prominent clinical features in social anxiety disorder. But it is self-obvious that the inclusion of the uncompassionate components in the SCS total score for a large part determines the relation between self-compassion and psychopathology, and hence obscures the true protective value of real compassionate self-responding (Muris 2016; Muris et al. 2016b). In one of our latest papers (Muris et al. 2019), we used Neff et al. (2018a, 2018b) own data to actually demonstrate that the relations between self-compassion and various indices of psychopathology symptoms were mainly carried by the uncompassionate components of self-judgement, isolation, and over-identification, but unfortunately Neff (2019) still finds arguments to discard these findings and to advocate the use of the SCS's total score as the most optimal index of self-compassion.

In agreement with what has been reported in previous studies (e.g., Neff and Vonk 2009), total self-compassion correlated moderately positively with self-esteem, indicating that they are distinct but related concepts (see also Muris et al. 2016a), with the most recent evidence suggesting that one's appraisal of worthiness (self-esteem) is an important antecedent of the capacity to extend compassion towards the self (self-compassion; Donald et al. 2018). When looking at the correlations between selfesteem and various SCS components, we noted equally strong positive associations with CSR (in particular self-kindness) and negative relations with USR (especially self-judgment and isolation), which means that self-esteem is linked to both compassionate and uncompassionate self-responding. The negative correlation between the total compassion score and neuroticism was also as anticipated (e.g., Geiger et al. 2018; Pfattheicher et al. 2017), but here further analysis indicated that in particular the USR components were responsible for this association, which of course fits well with the above described notion that self-judgement, isolation, and overidentification are more likely to be indicators of vulnerability and that their inclusion in the SCS pollutes the assessment of the protective construct of selfcompassion.

Self-esteem and neuroticism were also to some extent associated with adolescents' cognitive reactions to hypothetical daily life problems. More precisely, self-esteem was positively related to positive cognitive reactions and negatively to negative cognitive reactions, while neuroticism was mainly positively connected to negative cognitive reactions (cf. Carver and Conner-Smith 2010; Mullis and Chapman 2000). It is encouraging to note that selfcompassion accounted for unique variance in cognitive reactions scores, when controlling for the influence of neuroticism and self-esteem. This is particularly true for the independent positive contribution of CSR to positive cognitive reactions, a result suggesting that compassionate selfresponding as measured by the SCS indeed to some extent relates to more positive responses on a cognitive level when facing life stress and adversity, which is thought to be the mechanism underlying the adaptive function of (true) selfcompassion. Unique contributions of USR to positive and 
negative cognitive reactions were also found but seem less relevant in this context. This result indicates that uncompassionate self-responding colors thought processes in a negative way (Montero-Marin et al. 2016), which is unlikely to serve an adaptive purpose when young people have to deal with life problems (Flouri and Panourgia 2014).

An additional finding of this study was that boys displayed statistically significant higher total self-compassion scores on the SCS than girls, which is in line with Yarnell et al. (2015) meta-analysis. Further analysis of the gender difference in total self-compassion as found in the present study was not due to the fact that boys scored higher on the compassionate components, but rather that girls exhibited higher scores on the uncompassionate components of selfjudgment, isolation, and over-identification. We are not alone in this observation as other studies have also found that gender differences in self-compassion only exist on the negative subscales of the SCS (e.g., Bluth and Blanton 2014; Gill et al. 2018), and again this underlines the importance of making a distinction between CSR and USR (Muris et al. 2018). Other gender differences obtained in the present study corresponded well with what has been documented elsewhere in the literature. That is, girls displayed higher levels of negative cognitive reactions (Calvete and Cardenoso 2005) and neuroticism (Costa et al. 2001), while boys exhibited higher levels of general selfesteem (Skaalvik 1986).

It should be acknowledged that the present study suffers from a number of limitations. First and foremost, the crosssectional, correlational research design limits us from drawing conclusions on cause-effect relations. Although it is theoretically most plausible to interpret individual differences in self-compassion (presumably a trait) as the determinant of adolescents' negative and positive cognitive reactions (presumably more a state variable; Batts Allen and Leary 2010; Inwood and Ferrari 2018), we cannot rule out the possibility of a reverse relationship or a scenario in which self-compassion and cognitive reactions are both the product of a third variable. Second, the sample only consisted of adolescents who received pre-university education, which ensured us that the questionnaires were well understood and yielded valid responses. Nevertheless, it would be worthwhile to replicate the study in adolescents following lower levels of education in order to establish the generalizability of our findings. Third and finally, positive and negative reactions were assessed by means of the ARAQ, a scale that was construed for the purpose of the present study. While the scale proved to be reliable in terms of internal consistency and seems to possess good face and adequate factorial validity, its convergent validity with existing (cognitive) coping strategies measures or related constructs needs to be demonstrated. Future investigations could (also) make use of more validated instruments for assessing young people's thought processes and cognitive responses (such as the Children's Automatic Thoughts Scale; Schniering and Rapee 2002, or the Attributional Style Questionnaire for Adolescents; Rodriguez-Naranjo and Cano 2010) to adverse life events.

In spite of these shortcomings, the present study confirmed our main hypothesis that adolescents with higher levels of self-compassion will report higher levels of positive responses and lower levels of negative responses in times of adversity. Meanwhile, our results again raise questions on the appropriateness of using the total score of the SCS in this type of research. The compassionate and uncompassionate self-responding components included in this measure are clearly different in nature, with the former being more relevant if one is interested in protective mechanisms and the latter being more applicable within a context of vulnerability.

\section{Data Availability}

Data can be obtained via //ca-um-nas201/fpn_rdm \$/DM0728_PM_SelfCompassion under the map 07_Raw_data.

Acknowledgements Staff and students of Raayland College in Venray, The Netherlands, are kindly thanked for their participation in this study.

Author Contributions PM, HO, and CM designed the study, analyzed the data, and wrote the manuscript. $\mathrm{AH}$ and $\mathrm{MvdH}$ collected the data for the study and were also involved in the analysis of the data as well as the writing process.

\section{Compliance with Ethical Standards}

Conflict of Interest The authors declare that they have no conflict of interest.

Ethical Approval All procedures were in accordance with ethical standards. The research protocol was approved by the Ethical Review Committee Psychology and Neuroscience at Maastricht University, The Netherlands.

Informed Consent Informed consent was obtained from all individual participants included in the study.

Publisher's note: Springer Nature remains neutral with regard to jurisdictional claims in published maps and institutional affiliations.

Open Access This article is distributed under the terms of the Creative Commons Attribution 4.0 International License (http://crea tivecommons.org/licenses/by/4.0/), which permits use, duplication, adaptation, distribution, and reproduction in any medium or format, as long as you give appropriate credit to the original author(s) and the source, provide a link to the Creative Commons license, and indicate if changes were made. 


\section{References}

Astor-Dubin, L., \& Hammen, C. (1984). Cognitive versus behavioral coping responses of men and women: A brief report. Cognitive Therapy and Research, 8, 85-89.

Ayers, T. S., Sandler, I. N., West, S. G., \& Roosa, M. W. (1996). A dispositional and situational assessment of children's coping: Testing alternative models of coping. Journal of Personality, 64, 923-958.

Barbaranelli, C., Caprara, G. V., Rabasca, A., \& Pastorelli, C. (2003). A questionnaire for measuring the Big Five in late childhood. Personality and Individual Differences, 34, 645-664.

Batts Allen, A., \& Leary, M. R. (2010). Self-compassion, stress, and coping. Social and Personality Psychology Compass, 4, 107-118.

Bengtsson, H., Söderström, M., \& Terjestam, Y. (2016). The structure and development of dispositional compassion in early adolescence. Journal of Early Adolescence, 36, 840-873.

Bluth, K., \& Blanton, P. (2014). Mindfulness and self-compassion: Exploring pathways of adolescent wellbeing. Journal of Child and Family Studies, 23, 1298-1309.

Bluth, K., Campo, R. A., Futch, W. S., \& Gaylord, S. A. (2017). Age and gender differences in the associations of self-compassion and emotional well-being in a large adolescent sample. Journal of Youth and Adolescence, 46, 840-853.

Brenner, R. E., Heath, P. J., Vogel, D. L., \& Credé, M. (2017). Two is more valid than one: Examining the factor structure of the SelfCompassion Scale (SCS). Journal of Counseling Psychology, 64, 696-707.

Brenner, R. E., Vogel, D. L., Lannin, D. G., Engel, K. E., Seidman, A. J., \& Heath, P. J. (2018). Do self-compassion and self-coldness distinctly relate to distress and well-being? A theoretical model of self-relating. Journal of Counseling Psychology, 65, 346-357.

Byrne, D. G., \& Mazanov, J. (2002). Sources of stress in Australian adolescents: Factor structure and stability over time. Stress and Health, 18, 185-192.

Calvete, E., \& Cardenoso, O. (2005). Gender differences in cognitive vulnerability to depression and behavior problems in adolescents. Journal of Abnormal Child Psychology, 33, 179-192.

Carver, C. S., \& Conner-Smith, J. (2010). Personality and coping. Annual Review of Psychology, 61, 679-704.

Coroiu, A., Kwakkenbos, L., Moran, C., Thombs, B., Albani, C., Bourkas, S., Zenger, M., Brahler, E., \& Körner, A. (2018). Structural validation of the Swelf-Compassion Scale with a German general population sample. PLOS One, 13, e0190771.

Costa, J., Maroco, J., Pinto-Gouveia, J., Ferreira, C., \& Castilho, P. (2016). Validation of the psychometric properties of the SelfCompassion Scale. Testing the factorial validity and factorial invariance of the measure among borderline personality disorder, anxiety disorder, eating disorder, and general populations. Clinical Psychology and Psychotherapy, 23, 460-468.

Costa, P. T., Terracciano, A., \& McCrae, R. R. (2001). Gender differences in personality traits across cultures: Robust and surprising findings. Journal of Personality and Social Psychology, 81, 322-331.

Diedrich, A., Burger, J., Kirchner, M., \& Berking, M. (2017). Adaptive emotion regulation mediates the relationship between selfcompassion and depression in individuals with unipolar depression. Psychology and Psychotherapy: Therapy, Research and Practice, 90, 247-263.

Donald, J. N., Ciarrochi, J., Parker, P. D., Sahdra, B. K., Marshall, S. L., \& Guo, J. (2018). A worthy self is a caring self: Examining the developmental relations between self-esteem and selfcompassion in adolescents. Journal of Personality, 86, 619-630.

Ebata, A., \& Moos, R. (1991). Coping and adjustment in distressed and healthy adolescents. Journal of Applied Developmental Psychology, 12, 33-54.
Flouri, E., \& Panourgia, C. (2014). Negative automatic thoughts and emotional and behavioural problems in adolescence. Child and Adolescent Mental Health, 19, 46-51.

Geiger, M., Pfattheicher, S., Hartung, J., Weiss, S., Schindler, S., \& Wilhelm, O. (2018). Self-compassion as a facet of neuroticism? A reply to the comments of Neff, Toth-Kiraly, and Colosimo (2018). European Journal of Personality, 32, 393-404.

Gilbert, P. (2000). Social mentalities: Internal 'social' conflict and the role of inner warmth and compassion in cognitive therapy. In P. Gilbert \& K. G. Bailey(Eds.), Genes on the couch: Exploration in evolutionary psychotherapy (pp. 118-150). Hove, UK: Psychology Press.

Gilbert, P. (2005). ). Compassion: Conceptualizations, research, and use in psychotherapy. London, UK: Routledge.

Gill, C., Watson, L., Williams, C., \& Chan, S. W. Y. (2018). Social anxiety and self-compassion in adolescents. Journal of Adolescence, 69, 163-174.

Hampel, P., \& Petermann, F. (2005). Age and gender differences on coping in children and adolescents. Journal of Youth and Adolescence, 34, 73-83.

Hankin, B. L., Abramson, L. Y., Moffitt, T. E., Silva, P. A., McGee, R., \& Angell, K. E. (1998). Development of depression from preadolescence to young adulthood: Emerging gender differences in a 10-year longitudinal study. Journal of Abnormal Psychology, 107, 128-140.

Harter, S. (1999). The construction of the self: A developmental perspective. New York, NY: Guilford.

Harter, S. (1985). Manual for the Self-Perception Profile for Children. Denver: University of Denver.

Hogendoorn, S., Wolters, L. H., Vervoort, L., Prins, P. J. M., Boer, F., Kooij, E., \& De Haan, E. (2010). Measuring negative and positive thoughts in children: An adaptation of the Children's Automatic Thoughts Scale (CATS). Cognitive Therapy and Research, $34,467-478$.

Inwood, E., \& Ferrari, M. (2018). Mechanisms of change in the relationship between self-compassion, emotion regulation, and mental health. Applied Psychology: Health and Well-being, 10, 215-235.

Kopala-Sibley, D. C., Zuroff, D. C., Russell, J. J., \& Moskowitz, D. S. (2013). Understanding heterogeneity in social anxiety disorder: Dependency and self-criticism moderate fear responses to interpersonal cues. British Journal of Clinical Psychology, 53, $141-156$.

Leary, M. R., Tate, E. B., Adams, C. E., Batts Allen, A., \& Hancock, J. (2007). Self-compassion and reactions to unpleasant self-relevant events: The implications of treating oneself kindly. Journal of Personality and Social Psychology, 92, 887-904.

Lewinsohn, P. M., Gotlib, I. H., Lewinsohn, M., Seeley, J. R., \& Allen, N. B. (1998). Gender differences in anxiety disorders and anxiety symptoms in adolescents. Journal of Abnormal Psychology, 107, 109-117.

Lim, M. H., Rodebaugh, T. L., Zyphur, M. J., \& Gleeson, J. F. M. (2016). Loneliness over time: The crucial role of social anxiety. Journal of Abnormal Psychology, 125, 620-630.

Lopez, A., Sanderman, R., Ranchor, A., \& Schroevers, M. J. (2018). Compassion for others and self-compassion: Levels, correlates, and relationship with psychological well-being. Mindfulness, 9, $325-331$.

Lopez, A., Sanderman, R., Smink, A., Zhang, Y., Van Sonderen, E., Ranchor, A., \& Schroevers, M. J. (2015). A reconsideration of the Self-Compassion Scale's total score: Self-compassion versus selfcriticism. PLoS ONE, 10, e0132940.

MacBeth, A., \& Gumley, A. (2012). Exploring compassion: A metaanalysis of the association between self-compassion and psychopathology. Clinical Psychology Review, 32, 545-552. 
Marsh, I. C., Chan, S. W. Y., \& MacBeth, A. (2018). Self-compassion and psychological distress in adolescents - A meta-analysis. Mindfulness, 9, 1011-1027.

Montero-Marin, J., Gaete, J., Demarzo, M., Rodero, B., Serrano Lopez, L. C., \& Garcia-Campayo, J. (2016). Self-criticism: A measure of uncompassionate behaviors toward the self, based on the negative components of the Self-Compassion Scale. Frontiers in Psychology, 7, 1281.

Mullis, R. L., \& Chapman, P. (2000). Age, gender, and self-esteem differences in adolescent coping styles. Journal of Social Psychology, 140, 539-541.

Muris, P. (2016). A protective factor against mental health problems in youths? A critical note on the assessment of self-compassion. Journal of Child and Family Studies, 25, 1461-1465.

Muris, P., Mayer, B., Reinders, E., \& Wesenhagen, C. (2011). Personrelated protective and vulnerability factors of psychopathology symptoms in non-clinical adolescents. Community Mental Health Journal, 47, 47-60.

Muris, P., Meesters, C., \& Diederen, R. (2005). Psychometric properties of the Big Five Questionnaire for Children (BFQ-C) in a Dutch sample of young adolescents. Personality and Individual Differences, 38, 1757-1769.

Muris, P., Meesters, C., \& Fijen, P. (2003). The Self-Perception Profile for Children: Further evidence for its factor structure, reliability, and validity. Personality and Individual Differences, 35, 1791-1802.

Muris, P., Meesters, C., Pierik, A., \& De Kock, B. (2016a). Good for the self: Self-compassion and other self-related constructs in relation to symptoms of anxiety and depression in non-clinical youths. Journal of Child and Family Studies, 25, 607-617.

Muris, P., Otgaar, H., \& Petrocchi, N. (2016b). Protection as the mirror image of psychopathology: Further critical notes on the SelfCompassion Scale. Mindfulness, 7, 787-790.

Muris, P., Otgaar, H., \& Pfattheicher, S. (2019). Stripping the forest from the rotten trees: Compassionate self-responding is a way of coping, but reduced uncompassionate self-responding mainly reflects psychopathology. Mindfulness, 10, 196-199.

Muris, P., \& Petrocchi, N. (2017). Protection or vulnerability? A metaanalysis of the relations between the positive and negative components of self-compassion and psychopathology. Clinical Psychology and Psychotherapy, 24, 373-383.

Muris, P., Van den Broek, M., Otgaar, H., Oudenhoven, I., \& Lennartz, J. (2018). Good and bad sides of self-compassion: A face validity check of the Self-Compassion Scale and an investigation of its relations to coping and emotional symptoms in non-clinical adolescents. Journal of Child and Family Studies, 27, 2411-2421.

Neff, K. D. (2003a). Development and validation of a scale to measure self-compassion. Self and Identity, 2, 223-250.

Neff, K. D. (2003b). Self-compassion: An alternative conceptualization of a healthy attitude toward oneself. Self and Identity, 2, $85-101$

Neff, K. D. (2009). Self-compassion. In M. R. Leary \& R. H. Hoyle (Eds.), Handbook of individual differences in social behavior (pp. 561-573). New York, NY: Guilford Press.

Neff, K. D. (2016). The Self-Compassion Scale is a valid and theoretically coherent measure of self-compassion. Mindfulness, 7 , 264-274.

Neff, K. D. (2019). Setting the record straight about the SelfCompassion Scale. Mindfulness, 10, 200-202.

Neff, K. D., Long, P., Knox, M. C., Davidson, O., Kuchar, A., Costigan, A., Williamson, Z., Rohleder, N., Tóth-Király, I., \& Breines, J. G. (2018a). The forest and the trees: Examining the association of self-compassion and its positive and negative components with psychological functioning. Self and Identity, 17, 627-645.
Neff, K. D., Toth-Kiraly, I., \& Colosimo, K. (2018b). Self-compassion is best measured as a global construct and is overlapping with but distinct from neuroticism: A response to Pfattheicher, Geiger, Hartung, Weiss, and Schindler (2017). European Journal of Personality, 32, 371-392.

Neff, K. D., \& Vonk, R. (2009). Self-compassion versus global selfworth: Two different ways of relating to oneself. Journal of Personality, 77, 23-50.

Neff, K. D., Whittaker, T. A., \& Karl, A. (2017). Examining the factor structure of the Self-Compassion Scale in four distinct populations: Is the use of a total score justified? Journal of Personality Assessment, 99, 596-607.

O’Brien, M., Bahadur, M. A., Gee, C., Balto, K., \& Erber, S. (1997). Child exposure to marital conflict and child coping responses as predictors of child adjustment. Cognitive Therapy and Research, $21,39-59$.

Ormel, J., Rosmalen, J., \& Farmer, A. (2004). Neuroticism: A noninformative marker of vulnerability to psychopathology. Social Psychiatry and Psychiatric Epidemiology, 39, 906-912.

Penney, E. S., \& Abbott, M. J. (2014). Anticipatory and post-event rumination in social anxiety disorder: A review of the theoretical and empirical literature. Behaviour Change, 31, 79-101.

Pfattheicher, S., Geiger, M., Hartung, J., Weiss, S., \& Schindler, S. (2017). Old wine in new bottles? The case of self-compassion and neuroticism. European Journal of Personality, 31, 160-169.

Phelps, S., \& Jarvis, P. (1994). Coping in adolescence: Empirical evidence for a theoretically based approach to assessing coping. Journal of Youth and Adolescence, 23, 359-371.

Raes, F., Pommier, E., Neff, K. D., \& Van Gucht, D. (2011). Construction and factorial validation of a Short Form of the SelfCompassion Scale. Clinical Psychology and Psychotherapy, 18, 250-255.

Rodriguez-Naranjo, C., \& Cano, A. (2010). Development and validation of an Attributional Style Questionnaire for Adolescents. Psychological Assessment, 22, 837-851.

Schniering, C. A., \& Rapee, R. M. (2002). Development and validation of a measure of children's automatic thoughts: The Children's Automatic Thoughts Scale. Behaviour Research and Therapy, 40, 1091-1109.

Sirois, F. M., Molnar, D. S., \& Hirsch, J. K. (2015). Self-compassion, stress, and coping in the context of chronic illness. Self and Identity, 14, 334-347.

Skaalvik, E. M. (1986). Sex differences in global self-esteem: A research review. Scandinavian Journal of Educational Research, 30, 1971-1992.

Sutton, E., Schonert-Reichl, K. A., Wu, A. D., \& Stewart Lawlor, M. (2018). Evaluating the reliability and validity of the SelfCompassion Scale short form adapted for children ages 8-12. Child Indicators Research, 11, 1217-1236.

Williams, M. J., Dalgleish, T., Karl, A., \& Kuyken, W. (2014). Examining the factor structures of the Five Facet Mindfulness Questionnaire and the Self-Compassion Scale. Psychological Assessment, 26, 407-418.

Wright, M., Creed, P., \& Zimmer-Gembeck, M. J. (2010). The development and initial validation of a brief daily hassles scale suitable for use with adolescents. European Journal of Psychological Assessment, 26, 220-226.

Yarnell, L. M., Stafford, R. E., Neff, K. D., Reilly, E. D., Knox, M. C., \& Mullarkey, M. (2015). Meta-analysis of gender differences in self-compassion. Self and Identity, 14, 499-520.

Zessin, U., Dickhäuser, O., \& Garbade, S. (2015). The relationship between self-compassion and well-being: A meta-analysis. Applied Psychology: Health and Well-being, 7, 340-364. 